\title{
Retrieval lesson learned from NAST-I hyperspectral data
}

\author{
Daniel K. Zhou ${ }^{*}$,William L. Smith ${ }^{\ddagger}$ Xu Liu ${ }^{*}$, Allen M. Larar ${ }^{*}$, and Stephen A. Mango ${ }^{\dagger}$ \\ *NASA Langley Research Center, Hampton, VA 23681, USA \\ ${ }^{*}$ Hampton University, Hampton, VA 23668, USA \\ ${ }^{\dagger}$ NPOESS Integrated Program Office, Silver Spring, MD 20910, USA \\ daniel.k.zhou@nasa.gov
}

\begin{abstract}
The retrieval lesson learned is important to many current and future hyperspectral remote sensors. Validated retrieval algorithms demonstrate the advancement of hyperspectral remote sensing capabilities to be achieved with current and future satellite instruments.

OCIS codes: (010.1280) Atmospheric composition; (280.0280) Remote sensing
\end{abstract}

\section{Introduction}

The NPOESS (National Polar-orbiting Operational Environmental Satellite System) Airborne Sounder Testbed Interferometer (NAST-I) is designed to support the development of future satellite temperature and moisture sounders. The NAST was developed in 1998 and it has been collecting data through out numerous field campaigns (Smith et al., 1999). The methodology for thermodynamic parameters retrieved under cloud-free conditions was developed and validated; the algorithm was used to demonstrate that an improved moisture profile is retrieved from hyperspectral radiances (Zhou et al., 2002). Accurate retrieval of the surface properties (i.e., surface emissivity and temperature) is simultaneously achieved in order to obtained accurate terrestrial boundary layer (TBL) moisture. In addition to thermodynamics parameters retrieved, atmospheric trace gas (e.g., CO) retrieval is critical in air quality observation, modeling, and forecasting. NAST-I CO retrieval algorithm was developed to demonstrate the retrieval sensitivity and accuracy of this type of hyperspectral sounders under cloud-free conditions (Zhou et al., 2005; 2007a). The globe hosts many vast cloudy regions and a great deal of effort has gone into cloud detection and cloud-clearing processes (Smith et al. 2004). Nevertheless, the schemes dealing with cloud detection and cloud-clearing (Smith 1968) remain a major source of error in the final retrieval products. Recently, fast molecular and cloud transmittance models (Yang et al., 2001) have been used to enable the infrared radiances to be used under cloudy conditions with the accuracy required for sounding retrieval processing (Zhou et al., 2005; 2006). With this recently developed algorithm, cloud parameters as well as atmospheric profiles are retrieved simultaneously from infrared spectral radiance observations. A large amount of samples, retrieved and validated, are used to demonstrate a state-of-art retrieval algorithm based on the NAST-I hyperspectral data and how well this algorithm applies to the AIRS data. Experiences with the NAST-I and AIRS retrieval put us in a position of confidence that accurate retrievals will be obtained from future satellite hyperspectral instruments such as the IASI, the CrIS, the GIFTS, and the HES.

\section{Retrieval Demonstration}

High-resolution infrared radiance spectra obtained from near nadir observations provide atmospheric, surface, and cloud property information. A fast radiative transfer model, including cloud effects, is used for atmospheric profile and cloud parameter retrieval. The retrieval algorithm is presented along with its application to field experiment data from the NPOESS Airborne Sounder Testbed - Interferometer (NAST-I). The retrieval accuracy dependence on cloud properties is discussed. The initial EOF regression has laid a first step in dealing with infrared sounding data under cloudy conditions which is now significantly improved by the physical iteration inversion described in this study. Results achieved with airborne NAST-I observations show that accuracies close to those achieved in totally cloud-free conditions can be achieved down to cloud top levels. The accuracy of the profile retrieved below cloud top level is dependent upon the optical thickness and fractional coverage of the clouds. Retrieval accuracy of temperature and moisture profiles is greatly improved by the physical inversion. Details can be found elsewhere (Smith et al., 2005; Zhou et al., 2005; Zhou et al., 2006). The physical retrieval results of cloud and thermodynamic parameters are shown in Figure 1 (after Zhou et al., 2006). Results achieved with airborne NAST-I observations show that accuracies close to those achieved in totally cloud-free conditions can be achieved down to cloud top levels. The accuracy of the profile retrieved below cloud top level is dependent upon the optical thickness and fractional coverage of the clouds. Retrieval accuracy of temperature and moisture profiles is greatly improved by the physical inversion as shown by dropsonde validation. Therefore, the radiances can be accurately simulated by using physically inverted results which is important for direct assimilation into a forecast model. 

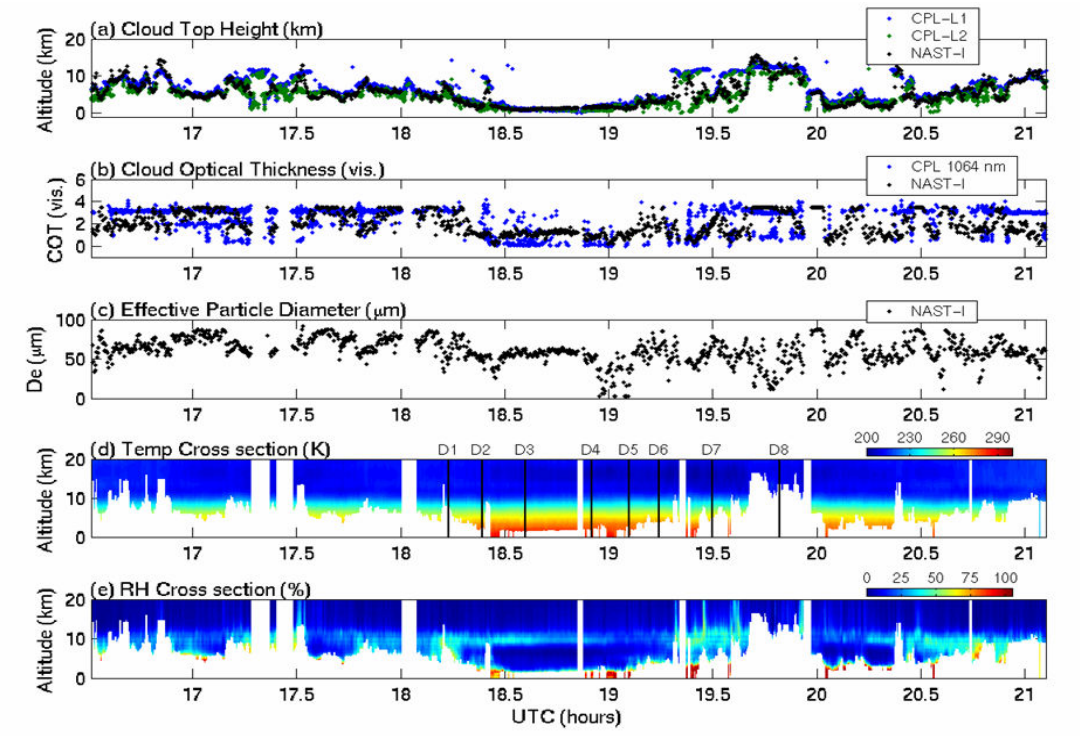

Fig. 1. Panel (a) plots NAST-I physically retrieved cloud top height compared with the CPL measured cloud top heights of the top 2 layers (L1 and L2). Panel (b) plots NAST-I retrieved visible cloud optical thickness (COT) compared with the CPL measurement. Panel (c) plots NAST-I retrieved cloud particle size. Panels (d) and (e) plot NAST-I physically retrieved temperature and relative humidity vertical cross sections, respectively. The areas wiped off are under the top layer of clouds where the cloud visible optical thickness is larger than one and under the lower "opaque" cloud. The black vertical bars in panel (d) indicate dropsonde locations.

The NAST-I field campaign data are also used to provide radiometric measurements including tropospheric trace species such as carbon monoxide (CO). The NAST-I CO inversion scheme is combined with a three-step procedure: (1) EOF (i.e., empirical orthogonal function) regression retrieval, (2) simultaneous matrix inversion, and (3) CO profile enhancement inversion. The NAST-I tropospheric CO retrieval algorithm has been developed to retrieve CO from NAST-I radiances and to investigate tropospheric $\mathrm{CO}$ vertical profile retrieval accuracy from a satellite ultraspectral sounder. The $\mathrm{CO}$ evaluation study having co-incident radiance and in-situ measurement data sets, enables an understanding of the accuracy of our current $\mathrm{CO}$ retrieval algorithm and validates the results based on theoretical simulations (Zhou et al., 2005). An example of CO retrieval from the EAQUATE shown in figure 2 (after Zhou et al., 2007a) indicates that the CO first guess profile plays a major role in the $\mathrm{CO}$ profile accuracy. The TBL CO accuracy, mainly determined by the first guess, affects the free tropospheric $\mathrm{CO}$ retrieval. However, $\mathrm{CO}$ variations in the free troposphere can still be captured while $\mathrm{CO}$ amount variations in the TBL are not retrieved very well. Thus, it is a challenge to obtain accurate CO profiles (especially in the TBL) from remote sounders such as NAST-I.
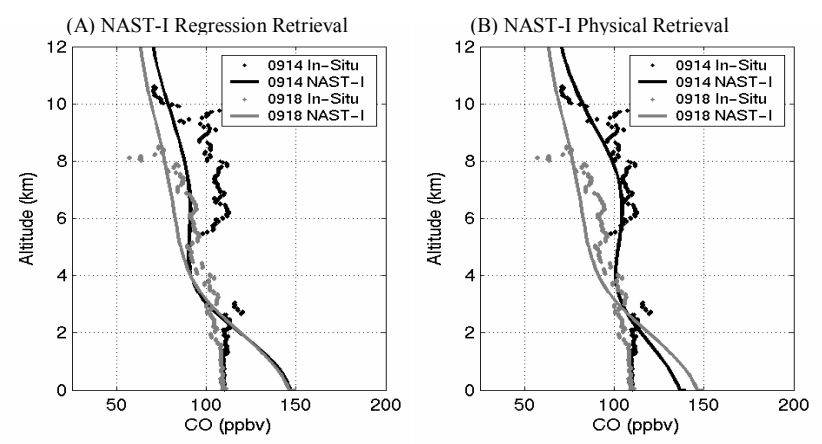

Fig. 2. Date-to-date CO profile inter-comparison: (A) NAST-I regression vs. in-situ, (B) NAST-I final physical retrieval vs. in-situ. Data from 14 September 2004 is plotted in gray while 18 September 2004 is plotted in black. In-situ data plotted in dots, NAST-I local mean in solid curves.

The Atmospheric InfraRed Sounder (AIRS) on the Earth Observing System (EOS) Aqua satellite was launched on 4 May 2002. Experience with NAST-I greatly helps the development of AIRS satellite data retrieval. The NAST retrieval algorithm has been used for the AIRS team algorithm validation (Zhou et al., 2007b). As the satellite data covers a large variety of surface and atmospheric conditions, the retrieval scheme is tested with a large variety of conditions. Initial study with AIRS data has been performed and more detailed validation over the land will be conducted. One granule of AIRS data (10 
September 2004; 01:00 UTC; local nighttime) shown in Figure 3 covers water and land including the vicinity of the Sahara Desert. The AIRS single field of view $(\sim 13.5 \mathrm{~km}$ at nadir) data are used; few cloudy spots were seen as the effective skin temperature contains cloud features (i.e., cooler "skin temperature"). However, this granule, for the most part, was collected under cloud-free conditions. The distribution of the surface emissivity images near $11 \mu \mathrm{m}$ and $8.15 \mu \mathrm{m}$ are plotted, capturing the feature of the surface emissivity variation. The spectacular features over the land, especially in the vicinity of the Sahara Desert, are clearly evident. Typical emissivity spectra retrieved from water, land, and desert are plotted in comparison with laboratory measurements. These reasonable retrieved surface properties greatly support the accurate atmospheric retrievals which are found elsewhere (Zhou et al., 2007b).

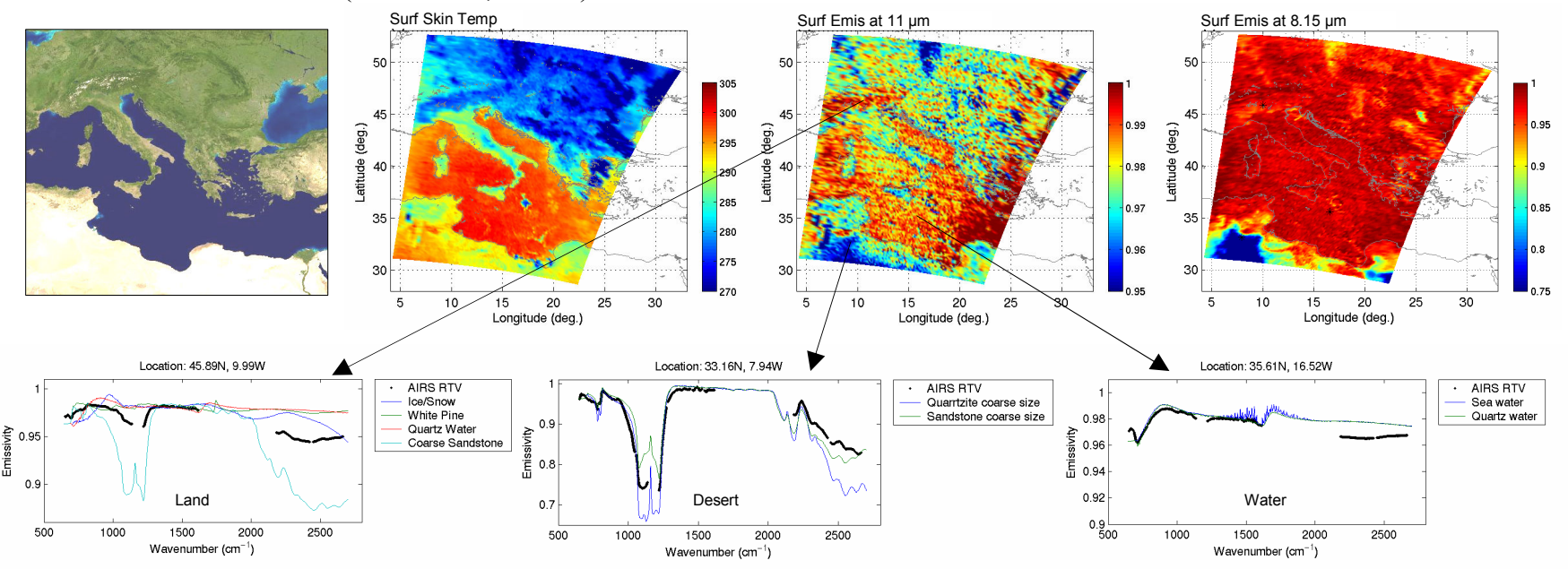

Fig. 3. Surface skin temperature and emissivity retrieved from the AIRS data, indicating a large variety of surface types is captured by the hyperspectral data and this inversion scheme.

\section{Conclusion}

The advantage of using NAST-I is that continuous spectrum of atmospheric radiation is obtained with a high spatial resolution as needed to develop retrieval algorithms. Lessons learned from the NAST program are beneficial to current and future satellite hyperspectral instruments such as AIRS, IASI, CrIS, GIFTS, and HES.

Acknowledgments: The NAST-I program is supported by the NPOESS Integrated Program Office, NASA Headquarters, and NASA Langley Research Center. The authors express their sincere thanks to the NAST-I team members from various organizations.

\section{References:}

Smith, W. L., 1968: An improved method for calculating tropospheric temperature and moisture from satellite radiometer measurements, Mon. Wea. Rev., 96, 387-396.

Smith, W. L., and co-authors, 1999: NAST-I: results from revolutionary aircraft sounding spectrometer, Proc. SPIE, 3756, $2-8$.

Smith, W. L., D. K. Zhou, H.-L. Huang, J. Li, X. Liu, and A. M. Larar, 2004: Extraction of profile information from cloud contaminated radiances, paper presented at the ECMWF Workshop on the Assimilation of High Spectral Resolution Sounders in NWP, June 28 July 1, 2004.

Smith, W. L., D. K. Zhou, A. M. Larar, S. A. Mango, H. B. Howell, R. O. Knuteson, H. E, Revercomb, and W. L. Smith Jr., 2005: The NPOESS Airborne Sounding Testbed Interferometer - remotely sensed surface and atmospheric conditions during CLAMS, J. Atmos. Sci., 62, 1117-1133.

Yang, P., B. C. Gao, B. A. Baum, Y. Hu, W. Wiscombe, S.-C. Tsay, D. M. Winker, S. L. Nasiri, 2001: Radiative Properties of cirrus clouds in the infrared (8-13 $\mu \mathrm{m})$ spectral region, J. Quant. Spectros. Radiat. Transfer, 70, 473-504.

Zhou, D. K., W. L. Smith, J. Li, H. B. Howell, G. W. Cantwell, A. M. Larar, R. O. Knuteson, D. C. Tobin, H. E. Revercomb, and S. A. Mango, 2002: Thermodynamic product retrieval methodology for NAST-I and validation, Appl. Opt., 41, 6957-6967.

Zhou, D. K., W. L. Smith, X. Liu, J. Li, A. M. Larar, and S. A. Mango, 2005a: Tropospheric CO observed with the NAST-I: retrieval methodology, analyses, and first results, Appl. Opt., 44, 3032-3044.

Zhou, D. K., W. L. Smith, X. Liu, A. M. Larar, H.-L. A. Huang, J. Li, M. J. McGill, and S. A. Mango, 2005b: Thermodynamic and cloud parameters retrieval using infrared spectral data, Geophys. Res. Lett., 32, L15805, doi:10.1029/2005GL023211.

Zhou, D. K., W. L. Smith, Sr., X. Liu, A. M. Larar, S. A. Mango, and H.-L. Huang, 2006: Physically-retrieving cloud and thermodynamic parameters from ultraspectral IR measurements, J. Atmos. Sci., in press (available upon request).

Zhou, D. K., A. M. Larar, X. Liu, G. W. Sachse, W. L. Smith, J. P. Taylor, S. M. Newman, and S. A. Mango, 2007a: NAST-I tropospheric CO retrieval validation during INTEX-NA and EAQUATE, submitted to the Q. J. R. Meteorol. Soc. (available upon request).

Zhou, D. K., and co-authors, 2007b, Retrieval validation during the European AQUA Thermodynamic Experiment, submitted to the $Q$. $J$. R. Meteorol. Soc. (available upon request). 